\title{
Feasibility of simultaneous PET-MR perfusion using a novel cardiac perfusion phantom
}

Jim O'Doherty ${ }^{1 *}$ D, Eva Sammut ${ }^{2,3}$, Paul Schleyer ${ }^{4}$, James Stirling ${ }^{1}$, Muhummad Sohaib Nazir ${ }^{2,5}$, Paul K. Marsden ${ }^{1}$ and Amedeo Chiribiri ${ }^{2,5}$

* Correspondence:

jim.odoherty@kcl.ac.uk

${ }^{1}$ Division of Imaging Sciences and Biomedical Engineering, PET Imaging Centre, King's College London, St. Thomas' Hospital, 1st Floor Lambeth Wing, St Thomas' Hospital, London SE1 7EH, UK Full list of author information is available at the end of the article

\begin{abstract}
Background: PET-MR scanners are beginning to be employed for quantitative myocardial perfusion imaging. In order to examine simultaneous perfusion calculations, this work describes a feasibility study of simultaneous PET-MR of gadolinium-based contrast agent (GBCA) and PET radiotracer in a novel cardiac perfusion phantom.

Results: $\left[^{18} \mathrm{~F}\right] \mathrm{F}^{-}$and GBCA were injected simultaneously into a cardiac phantom using a range of ground-truth myocardial perfusion rates of 1 to $5 \mathrm{ml} / \mathrm{g} / \mathrm{min}$. PET quantification of $K_{1}(\mathrm{ml} / \mathrm{g} / \mathrm{min})$ was performed using a single tissue compartment model. MR perfusion was calculated using a model-independent signal deconvolution technique. PET and MR signal traces from the phantom aorta and myocardial sections show true simultaneous PET and MR arterial input functions (AIF) and myocardial uptake respectively at each perfusion rate. Calculation of perfusion parameters showed both $K_{1}$ and $h(t=0)$ (PET and MR perfusion parameters respectively) to be linearly related with the ground truth perfusion rate $\left(P_{T}\right)$, and also linearly related to each other $\left(R^{2}=0.99\right)$. The highest difference in perfusion values between $K_{l}$ and $P_{T}$ was $16 \%$ at $1 \mathrm{ml} / \mathrm{g} / \mathrm{min}$, and the mean difference for all other perfusion rates was $<3 \%$.
\end{abstract}

Conclusions: The perfusion phantom allows accurate and reproducible simulation of the myocardial kinetics for simultaneous PET-MR imaging, and may find use in protocol design and development of PET-MR based quantification techniques and direct comparison of quantification of the two modalities.

Keywords: PET-MR, Cardiology, Perfusion, Flow

\section{Background}

Cardiac magnetic resonance (CMR) plays an increasing role in the diagnosis and stratification of patients with suspected coronary artery disease (CAD) justified by its high spatial resolution, tissue contrast and the ability to provide reproducible quantitative data on parameters such as left ventricular volumes and mass. CMR is also increasingly used to assess inducible ischaemia (Fihn et al., 2012; Task Force et al., 2013). In carefully controlled situations, CMR techniques have been shown to also provide absolute quantitative measurements of myocardial blood flow (MBF) and myocardial flow reserve (MFR) (Jerosch-Herold, 2010).

(c) The Author(s). 2017 Open Access This article is distributed under the terms of the Creative Commons Attribution 4.0 International License (http://creativecommons.org/licenses/by/4.0/), which permits unrestricted use, distribution, and reproduction in any medium, provided you give appropriate credit to the original author(s) and the source, provide a link to the Creative Commons license, and indicate if changes were made. 
Positron emission tomography-computed tomography (PET) imaging is a highly accurate method for assessment of obstructive coronary artery disease (CAD), with a sensitivity and specificity of approximately 90\% (Di Carli et al., 2007) and is considered the reference method for non-invasive quantification of myocardial perfusion (Bengel et al., 2009). Dynamic PET imaging can be performed using short-lived metabolized tracers (e.g. $\left[{ }^{82} \mathrm{Rb}\right] \mathrm{Cl},\left[{ }^{13} \mathrm{~N}\right] \mathrm{NH}_{3}$ ) or freely-diffusible tracers $\left(\right.$ e.g. ${ }^{15} \mathrm{O}-\mathrm{H}_{2} \mathrm{O}$ ) for quantification of absolute MBF and MFR.

The recent introduction of simultaneous hybrid PET-MR systems for combined molecular and functional imaging could be of great use in terms of understanding underlying cardiac pathophysiology and improving cross-modality validation. Multiple images comprising structural and functional information of the same tissue in the same physiological state can be acquired simultaneously. The combination of PET and MR acquisitions can provide further benefits in cardiac imaging such as real-time motion correction (Petibon et al., 2013), reduced patient scan time compared to independent CMR and PET-CT scans (Ratib \& Nkoulou, 2014), and a reduction in exposure to ionizing radiation (Ratib et al., 2013).

Due to the demanding technical requirements of first-pass perfusion imaging, the use of simultaneous PET-MR systems for quantitative cardiac imaging is only just emerging. Sequential CMR and PET perfusion measurements in a on the same day have shown that physiological variations in the time between studies (i.e. hemodynamic conditions) are a major factor (Morton et al., 2012). Recent work has performed simultaneous PET-MR in $\left[{ }^{18}\right.$ F]FDG cases to examine cardiac viability (Nensa \& Schlosser, 2014), cardiac sarcoma (Nensa et al., 2015) and active inflammation imaging of cardiac sarcoidosis (Schneider et al., 2014). MFR determined from $\left[{ }^{15} \mathrm{O}\right] \mathrm{H}_{2} \mathrm{O}$ PET from both PET-MR and PET-CT systems has been compared from 10 patients, detailing a high intra-class correlation coefficient of 0.98 (Kero et al., 2017). Another group studied the feasibility of acquiring MR and PET perfusion profiles simultaneously using dynamic contrast enhancement MR (DCE-MR) and $\left[{ }^{13} \mathrm{~N}\right] \mathrm{NH}_{3}$ PET for 10 patients, showing a correlation of $\mathrm{R}^{2}=0.67$ for rest and stress MBF and $\mathrm{R}^{2}=0.48$ for MFR (Zhang et al., 2013). A major confounding factor in the correlation between PET and MR perfusion comparison involves the difference in the tracer mechanism. Gadolinium based contrast agents (GBCA) do not undergo any intracellular processes, remaining distributed only within the extracellular space, whereas PET radiotracers typically enter and exit the myocyte. Thus there is also a lack of similarities between approaches to quantify perfusion on CMR and PET techniques such as modeling assumptions, fitting methods and parameter constraints (Gerber, 2012). Also of note in simultaneous imaging is the potential effects of contrast agent on the MR-based map for attenuation correction of PET sinograms (Rischpler et al., 2013; Rischpler et al., 2015).

There is thus room to improve correlation between PET and CMR perfusion quantification techniques, and a physiologically validated phantom with the capability of simultaneous PET-MR acquisition is likely to add to the growing body of knowledge. Our perfusion phantom has previously been validated to provide data suitable for quantitative analysis (Zarinabad et al., 2012) and has been employed in MR (Chiribiri et al., 2013) and CT quantification (Otton et al., 2013). Our work here follows on from our first investigations of simultaneous PET-MR phantom acquisitions using simultaneous injections of radiotracer and GBCA (O'Doherty et al., 2016). We 
aimed to investigate if perfusion estimates calculated independently via PET and MR techniques are related.

\section{Material and Methods}

\section{Phantom}

We used an in-house designed and built myocardial perfusion phantom, which has previously been described in detail (Chiribiri et al., 2013). Briefly, water is pumped through an MR-safe myocardial perfusion phantom placed in the scanner. The phantom is representative of the large thoracic vessels and of the heart of a $60 \mathrm{~kg}$ subject. It is composed of four cardiac chambers (120 ml each) and associated thoracic vessels (aorta, pulmonary artery, pulmonary vein, vena cava). A schematic representation detailing the phantom itself and supporting precision pumping and monitoring mechanisms is shown in Fig. 1. Myocardial perfusion is controlled in real time by flow meters continuously sampling the flow rate by means of highprecision digital flow meters (Atrato, Titan, Sherborne, United Kingdom) and providing re-adjustment of the speed of rotation of roller pumps through a feedback mechanism. Perfusion values were obtained by means of measurements of the distribution volume for the radioactive tracer and for the GBCA, and dividing the flow rate by this value. All pump controls and flow/perfusion rates are handled remotely from a custom-written LabVIEW application (LabVIEW Professional Development System 2014, National Instruments, Austin TX, USA) running on dedicated workstation and remotely controlled using an iPad application (Dashboard for LabVIEW, National Instruments, Austin TX, USA). As no radiotracer or GBCA re-entered the system after injection, we utilized a non-recirculating model in order to study first-pass myocardial perfusion measurements.

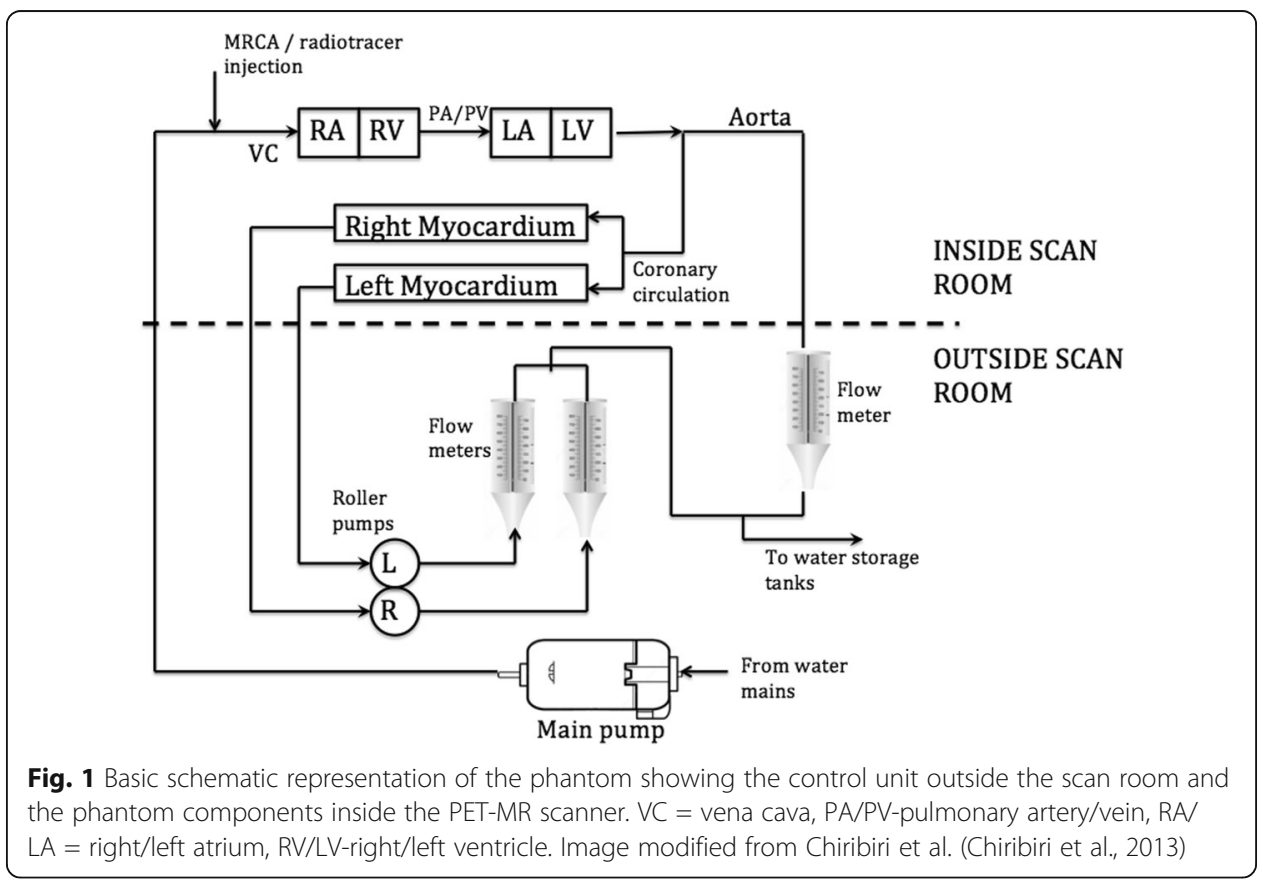




\section{Scanning parameters}

We performed PET-MR imaging on a 3 T Siemens Biograph mMR scanner (Siemens Healthcare $\mathrm{GmbH}$, Erlangen, Germany). The MR sequence consisted of a clinically utilized imaging protocol, namely a 2D TurboFLASH saturation recovery gradient echo sequence $\left(\mathrm{TE}=1 \mathrm{~ms}, \mathrm{TR}=164 \mathrm{~ms}\right.$, Flip angle $=10^{\circ}$, slice thickness $=6 \mathrm{~mm}$, pixel spacing $=1.875 \mathrm{~mm}$, matrix size $144 \times 192$ voxels, with temporal resolution of 1 image per cardiac beat). MR data were acquired in a single transverse plane identified by markings on the phantom, the locations of which correspond to a known dispersion volume for the GBCA and radiotracer. Cardiac output flow rate was set to $31 / \mathrm{min}$, with true myocardial perfusion rates (hereon denoted $P_{T}$ ) set to $1,2,3,4$ and $5 \mathrm{ml} / \mathrm{g} / \mathrm{min}$. A previously validated dual-bolus protocol was used for GBCA injection, with a pre-bolus of $0.001 \mathrm{mmol} / \mathrm{kg}$ of GBCA injected before a main bolus of $0.01 \mathrm{mmol} / \mathrm{kg}$ (Ishida et al., 2011). A minimum pause of $30 \mathrm{~s}$ was allowed between the pre-bolus and the main bolus of GBCA to ensure return of signal in the vascular and myocardial compartments to baseline values.

3D PET data were acquired in a single list-mode file and re-binned into short frames during the peak influx and washout phases $(60 \times 3 \mathrm{~s})$ and longer frames towards the end of the washout phase $(12 \times 15 \mathrm{~s})$. PET image frames were reconstructed using the standard manufacturer-issued filtered back-projection (FBP) algorithm available on the scanner $(344 \times 344$ matrix, Gaussian smoothing filter of $4 \mathrm{~mm}$. Resulting PET voxel sizes were $2.086 \mathrm{~mm} \times 2.086 \mathrm{~mm} \times 2.031 \mathrm{~mm}$. Attenuation correction of PET data was provided by the standard dual-point VIBE T1-weighted Dixon sequence available on the mMR scanner front end (Martinez-Moller et al., 2009). Total attenuation of the phantom is low as there is no attenuating material surrounding the phantom.

A mean injected activity of $207.8 \pm 9 \mathrm{MBq}$ was injected in order to exclude potential confounding dead-time effects in the PET detectors which has been shown to occur in clinical situations (O'Doherty et al., 2014; Renaud et al., 2016). After preloading of $\left[{ }^{18} \mathrm{~F}^{\mathrm{F}^{-}}\right.$into the tubing, the main bolus of GBCA (Gadovist ${ }^{\circ}$, Bayer HealthCare, Berlin, Germany) and $\left[{ }^{18} \mathrm{~F}\right] \mathrm{F}^{-}$were injected simultaneously via a contrast injection system (Spectris Solaris, Bayer AG, Leverkusen, Germany) through the vena cava tubing of the phantom (Fig. 1). Simultaneous dynamic PET-MR imaging was performed for a total of 300 s. A single simultaneous PET-MR acquisition was performed at each $P_{T}$ step, and each step was repeated for an estimate of repeatability of the phantom. After each scan, water was pumped through the myocardial compartments of the phantom for a minimum of $60 \mathrm{~s}$ between experiments to ensure a complete washout of GBCA and radiotracer before the next scan.

\section{Image analysis}

Dynamic PET images were analyzed in PMOD v 3.7 (PMOD Technologies, Zurich, Switzerland) to produce time-activity curves (TACs). 2D MR images were analyzed in OsiriX (OsiriX 64-bit, version 8.0.2, Pixmeo SARL, Geneva, Switzerland) to produce time-intensity curves (TICs). A region of interest (ROI) of $1.6 \mathrm{~cm}$ (tubing diameter) was placed over the aorta of the phantom, and ROIs of $4 \mathrm{~cm}$ diameter were placed over the left and right myocardial sections, carefully including the complete section of the vessel and tissue compartment in the segmentation. Positioning of ROIs on the PET 
image plane corresponding to the MR image plane was determined from fusion of the dynamic 3D PET and 2D summed dynamic MR images using PMOD. ROIs were placed on PET images over the same spatial extent as the MR ROIs. The PET volumes of interest (VOIs) were $6.093 \mathrm{~mm}$ thick (3 PET slices) in the axial-dimension in order to match the slice thickness of the MR data $(6 \mathrm{~mm})$. All PET data were decay-corrected to the scan start time. We thus produced a set of TACs and TICs for the aorta and myocardial compartments over the range of $P_{T}$.

\section{MR perfusion calculation}

In-house software was used for perfusion quantification (Labview 2014 for Mac, National Instruments, Austin, USA). A model-independent deconvolution approach was used to calculate the tissue impulse response function, providing results in units of $1 / \mathrm{s}$ (Patel et al., 2010) and was not scaled to be in units of $\mathrm{ml} / \mathrm{g} / \mathrm{min}$. Briefly, relative perfusion can be calculated based on the central volume principle using a signal deconvolution method (Jerosch-Herold et al., 1998). The TIC for the myocardial uptake function, $M(t)$, can be calculated from the TIC for the arterial input function, $C_{i n}(t)$, convolved with the tissue impulse response function $h(t)$ :

$$
M(t)=\int_{0}^{t} C_{\text {in }}(t-\tau) \cdot h(t) d t=\int_{0}^{t}\left[C_{\text {in }}(\tau)-C_{\text {out }}(\tau)\right] d \tau
$$

in which $C_{\text {out }}(t)$ denotes the contrast concentrations in the venous out-perfusion. We performed this calculation using the pre-bolus curve, $C_{i n}(t)$, as an input function, in order to minimize the effect of signal saturation by the main bolus of higher GBCA concentration, an effect which has been noted in previous work in patients and with this phantom at high GBCA dosages (Chiribiri et al., 2013; Ishida et al., 2011). In the range of physiological concentration used in the pre-bolus injection, MR signal intensity is proportional to GBCA concentration. The tissue impulse response function $h(t)$ has the shape of an exponential decay, and MR relative perfusion measurements were calculated from the $h(t))$ when $h(t=0)$, i.e. at the peak value of the exponential decay. The delay between the arterial input TIC and the myocardial TIC was accounted for in the model (Zarinabad et al., 2013).

\section{PET perfusion calculation}

PET data was modeled using a one-tissue compartment model characterized by a one blood compartment, one tissue compartment and two rate constants $K_{1}$ (uptake rate constant in units of $\mathrm{ml} / \mathrm{g} / \mathrm{min}$ ) and $k_{2}$ (clearance rate from tissue to blood constant in units of $\min ^{-1}$ ). For this phantom study, using $\left[{ }^{18} \mathrm{~F}\right] \mathrm{F}^{-}$we assume an extraction fraction of 1.0 due to the lack of any metabolic processes, and thus the $K_{1}$ constant is entirely representative of perfusion. In order to eliminate any prospective bias, PET and MR data were analyzed independently by two different authors blinded to the true myocardial perfusion rates, $P_{T}$ (PET analysis by JOD, MR analysis by AC).

The terms 'flow' and 'perfusion' have been used interchangeably in both PET and MR literature. Owing to the fact that rates of liquid through our phantom were calibrated in terms of $\mathrm{ml} / \mathrm{g} / \mathrm{min}$ (i.e. units of perfusion) and $K_{1}$ values from PET kinetic modeling were in the same units, we opt to keep consistency with terminology and use the term 'perfusion' rather than 'flow' (i.e. units of $\mathrm{ml} / \mathrm{min}$ ). 


\section{Results}

\section{Simultaneous imaging}

As the PET acquisition is fully 3D (25.8 cm field of view), all myocardial chambers can be visualized simultaneously. Figure 2 illustrates the rapid passage of radiotracer from the right atrium to ventricle, through the pulmonary circulation and into the left atrium and ventricle and exiting through the aorta. Figure 3 displays a fused transaxial image of the single MR slice with the corresponding merged 3 PET slices covering the same axial extent. The expected GBCA and PET radiotracer distribution through the phantom can be seen at increasing time points of the 2D MR imaging sequence and fused PET-MR images detailing the first pass dynamics of the phantom. The inset image of Fig. 3 shows the passage of MR contrast only, which temporally matches the distribution of PET radiotracer.

Image processing of the ROI/VOIs to produce TACs and TICs allows comparison of resulting mean PET kBq/ml to MR signal intensity during transit of the GBCA and radiotracer, as shown in Fig. 4. As the repeat injection of $\left[{ }^{18} \mathrm{~F}\right] \mathrm{F}^{-}$and $\mathrm{GBCA}$ was performed using the same timings and methodology as the first test, the time traces produced by both PET and MR data were similar. Although not shown in Fig. 4 for clarity, for a single $P_{T}$ of $4 \mathrm{ml} / \mathrm{g} / \mathrm{min}$, one standard deviation of the mean PET activity concentration from the VOI varied over time from \pm 2 to $\pm 24 \%$, while that of the MR mean ROI signal intensity varied over time from $\pm 13 \%$ to $\pm 29 \%$. Standard deviations were similar for other values of $P_{T}$. Figure 5 shows a comparison of the input functions from both imaging methodologies normalised by their respective maximum signal intensities, firstly between the main MR bolus peak of GBCA and the radiotracer (A), secondly between the MR prebolus peak (which was used for the MR perfusion analysis) and a time-shifted PET TAC overlaid to provide comparison (B), and finally a normalized comparison of the functions obtained from the myocardial chamber $(C)$. From these traces, it can be observed that the input functions for both PET and MR models show similar characteristics.
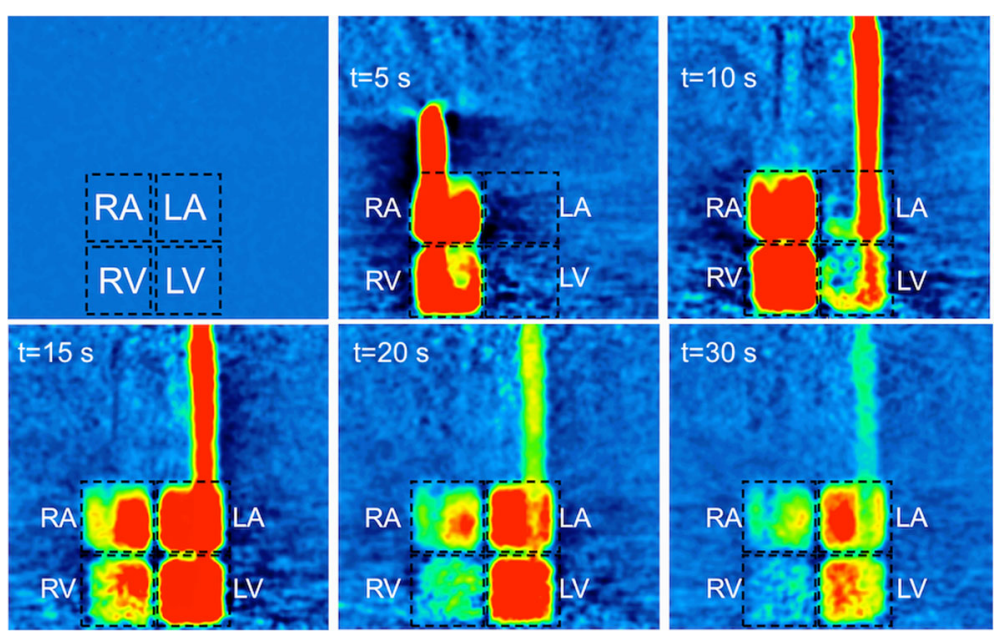

Fig. 2 Single coronal PET slice from the 3D phantom acquisition at a cardiac output rate of $3 \mathrm{~L} / \mathrm{min}$, showing an example of radiotracer distribution in the myocardial chambers at increasing post-injection time points. All images are shown at the same windowing and level. RA/LA = right/left atrium, RV/LV-right/left ventricle 

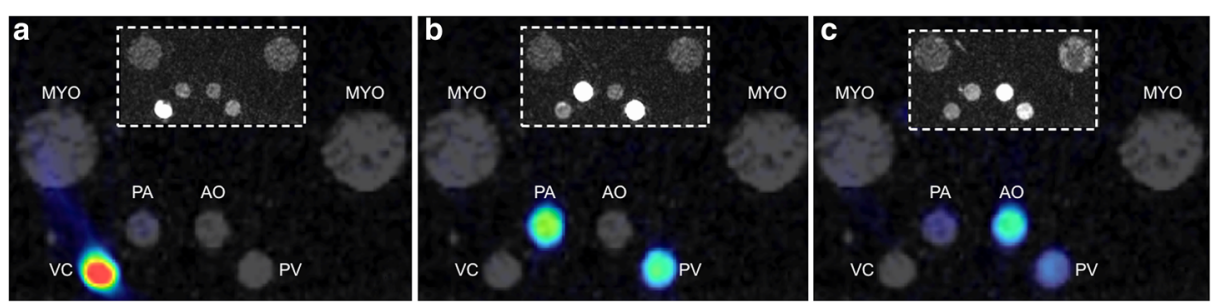

Fig. 3 Example fused PET-MR images showing dynamics of GBCA and radiotracer transfer through the phantom. $\mathbf{a}$ - bolus in the VC $(t=0 \mathrm{~s}) \cdot \mathbf{b}$ - outperfusion from the RV through the PA ( $t=3 \mathrm{~s}), \mathbf{c}$ - coronary circulation to the PV $(t=5 \mathrm{~s})$ and the aorta $(\mathrm{AO})$. Inset images show the time distribution of GBCA only

\section{Perfusion calculations}

PET datasets were used to calculate perfusion $(\mathrm{ml} / \mathrm{g} / \mathrm{min})$ via $K_{1}$, and $\mathrm{MR}$ datasets to calculate relative perfusion values via $h(t=0)$ as described above. Resulting $K_{1}$ and $h(t=0)$ and are shown in Table 1 . Figure 6 shows three plots detailing the relationship between $K_{1}$ and $P_{T}, h(t=0)$ and $P_{T}$ and also $h(t=0)$ and $K_{1}$. The results show that $K_{1}$ is linearly related to $P_{T}\left(\mathrm{R}^{2}=0.99\right)$, and that $h(t=0)$ is also linearly related to both $P_{T}$ and $K_{1}\left(R^{2}\right.$ values of 0.99 in both cases).

\section{Discussion}

We performed PET-MR tests using a specialized cardiac phantom allowing assessment of myocardial perfusion measurements with both imaging methodologies from simultaneously acquired data. Both PET and MR are accurate tools for the assessment of myocardial

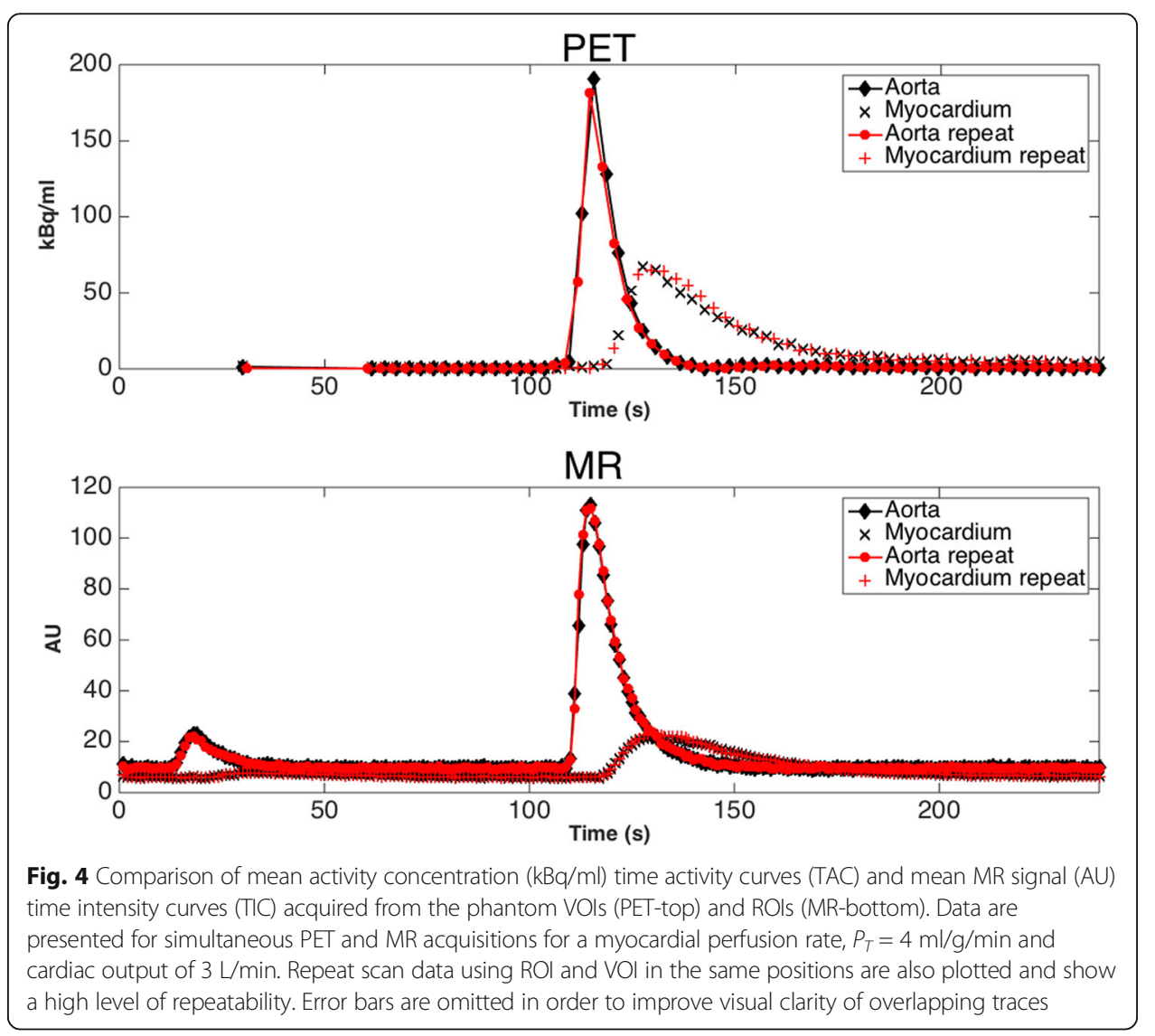




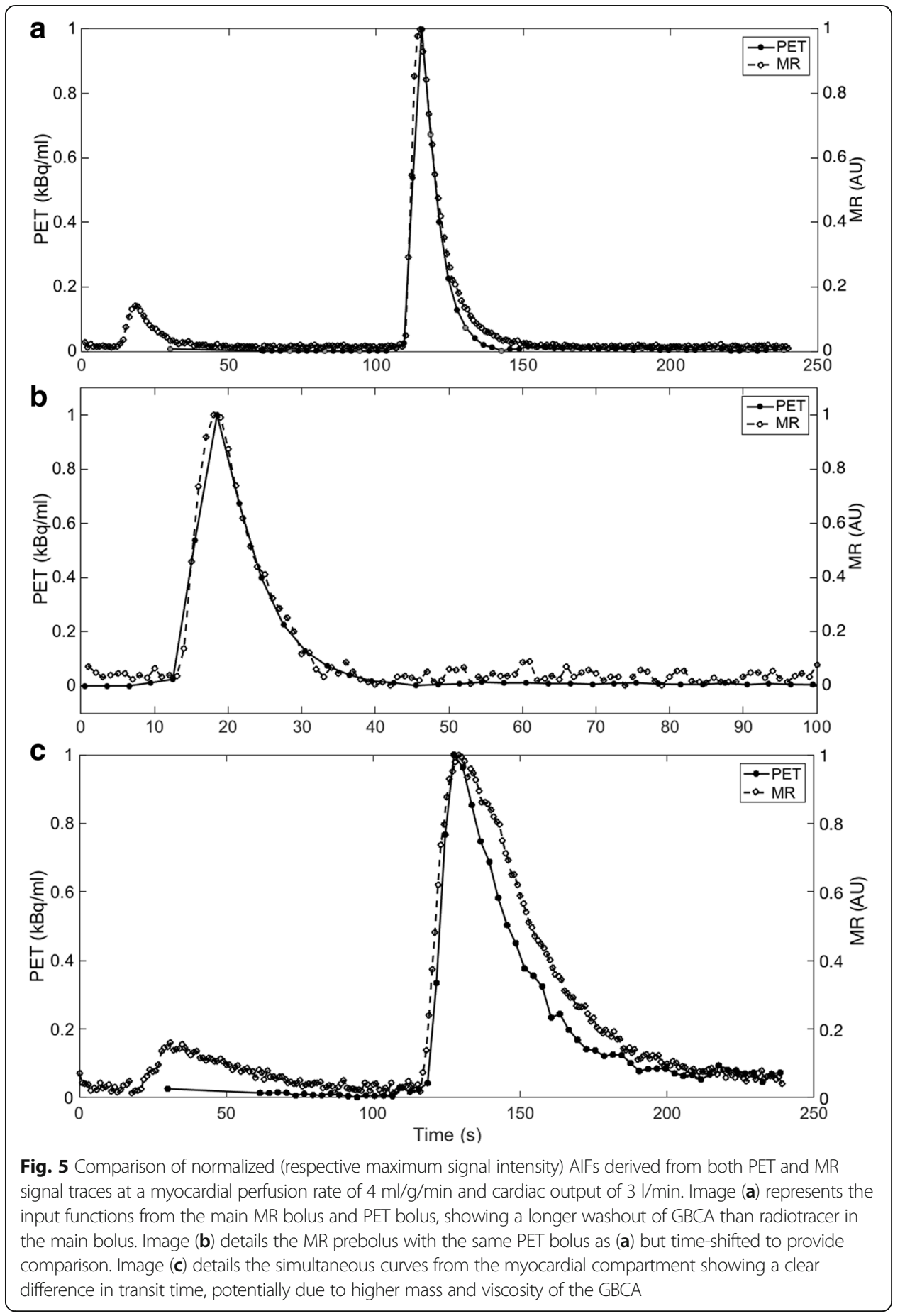

ischaemia, however there are drawbacks to each technique. For example in MR, derivation of fully quantitative perfusion units remain a complex process due to the relationship between signal intensity and gadolinium contrast and dependence on acquisition sequence (Jerosch-Herold, 2010), Also 2D imaging is favoured due to dynamic imaging meaning that perfusion calculations cannot be representative of the entire volume. In PET, the cost of the perfusion exam can be prohibitive and the procedure is based on access to short-lived radiotracers thus requiring access to a cyclotron. There is also a not insignificant radiation dose 
Table 1 Results of employing a one compartment kinetic model to the PET and deconvolution model to the MR data (Eq. 1)

\begin{tabular}{|c|c|c|c|c|c|c|}
\hline \multirow[b]{2}{*}{$\begin{array}{l}P_{T} \\
(\mathrm{ml} / \mathrm{g} / \mathrm{min})\end{array}$} & \multicolumn{4}{|l|}{ PET } & \multicolumn{2}{|l|}{$\underline{M R}$} \\
\hline & $\begin{array}{l}K_{1} \\
(\mathrm{ml} / \mathrm{g} / \mathrm{min})\end{array}$ & $\begin{array}{l}K_{1} \\
\text { SE (\%) }\end{array}$ & $\begin{array}{l}\mathrm{k}_{2} \\
(1 / \mathrm{min})\end{array}$ & $\begin{array}{l}k_{2} \\
S E(\%)\end{array}$ & $\begin{array}{l}h(t=0) \\
\left(s^{-1}\right)\end{array}$ & $\begin{array}{l}h(t=0) \\
\text { SE }(\%)\end{array}$ \\
\hline 1 & 1.14 & 3.8 & 0.96 & 7.83 & 0.132 & 4.019 \\
\hline 2 & 1.93 & 2 & 1.62 & 1.71 & 0.213 & 4.141 \\
\hline 3 & 2.94 & 1.55 & 2.44 & 2.05 & 0.279 & 3.346 \\
\hline 4 & 3.81 & 2.41 & 3.20 & 2.93 & 0.339 & 3.834 \\
\hline 5 & 5.14 & 1.83 & 4.43 & 3.35 & 0.403 & 2.985 \\
\hline \multicolumn{7}{|l|}{ REPEAT } \\
\hline 1 & 1.18 & 0.5 & 0.99 & 7.68 & 0.133 & 3.953 \\
\hline 2 & 2.07 & 1.87 & 1.87 & 1.74 & 0.201 & 3.112 \\
\hline 3 & 2.98 & 2.6 & 2.60 & 2.06 & 0.293 & 4.526 \\
\hline 4 & 3.98 & 3.21 & 3.21 & 2.16 & 0.384 & 4.259 \\
\hline 5 & 5.07 & 4.44 & 4.43 & 3.48 & 0.457 & 3.214 \\
\hline
\end{tabular}
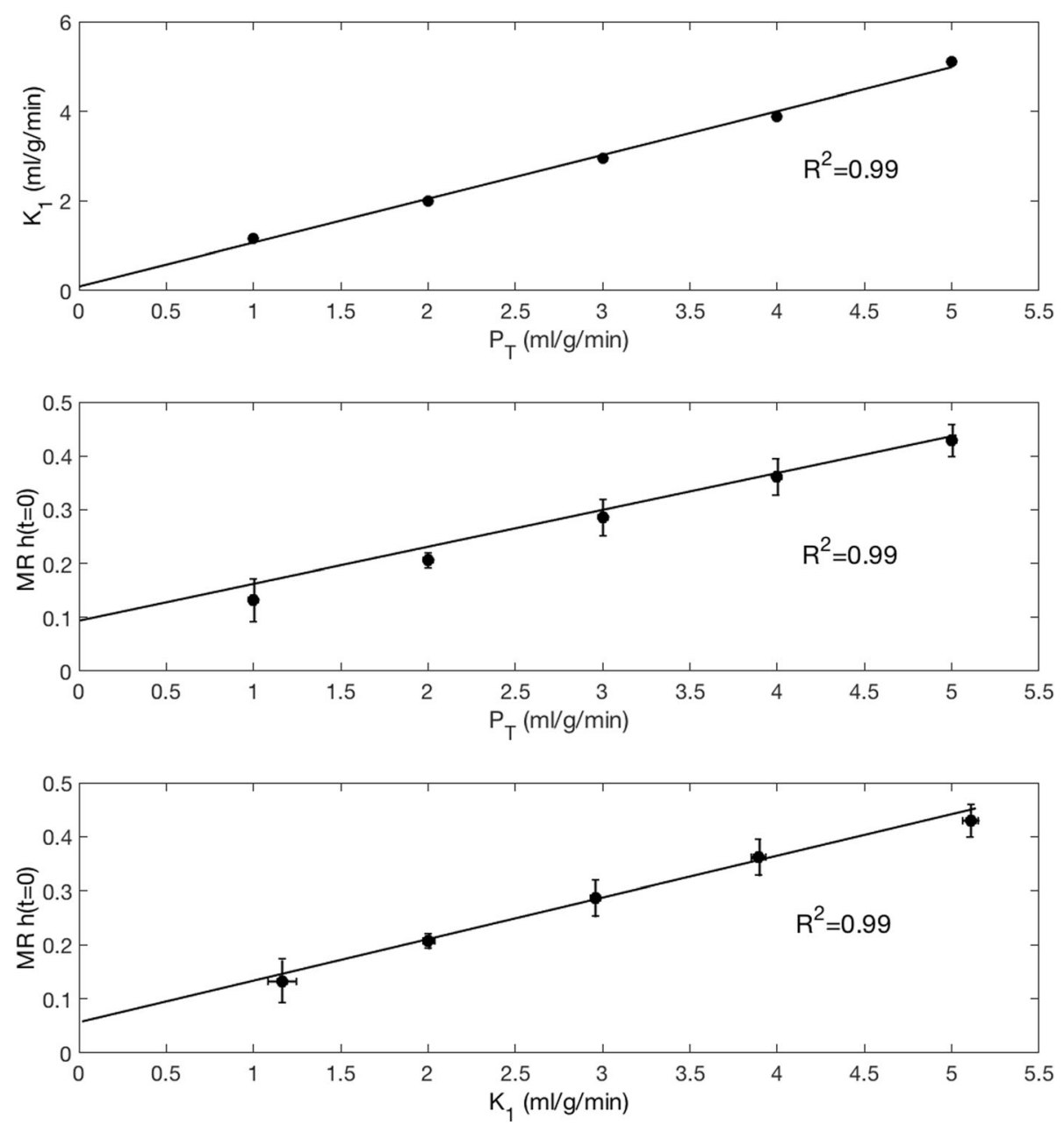

Fig. 6 Top - Resulting $K_{1}$ values from a single compartment model for PET data plotted against $P_{T}$. Middle MR values of $h(t=0)$ from model-independent deconvolution for MR images plotted against the range of $P_{T}$. Bottom - MR values of $h(t=0)$ plotted against the range of $K_{l}$ from PET kinetic modeling 
associated with the radiotracer. Recent work has investigated the complimentary information generated by simultaneous late gadolinium enhancement and 18F-FDG imaging (Rischpler et al., 2015). Although the principles of image formation between MR and PET are based entirely on different physical principles, we have shown that similar TACs and TICs from the arterial and myocardial compartments can be obtained from a single short acquisition. Furthermore, it has been possible to show that the results obtained with the employed MR and PET models are linearly related to the true myocardial perfusion rate, $P_{T}$.

The phantom is physiologically relevant and as such is able to explore some relevant aspects of perfusion dynamics of the human heart. Figures 2 and 3 demonstrate the distribution of both GBCA and radiotracer through the right side of the cardiac chambers followed by the left, with perfusion through the myocardial compartments following shortly after. Simultaneous traces of PET activity concentration and MR signal intensity in Fig. 4 demonstrate the transit of radiotracer and GBCA through the phantom, showing that true simultaneity of PET and MR signals can be achieved in this phantom. Our data also indicate that when a dual bolus approach is used in MR, linear perfusion estimates to those obtained in PET can be achieved.

In this study, we adopted a dual-bolus injection scheme previously described and validated by our group (Ishida et al., 2011; Schuster et al., 2013). The results of this study demonstrate that this approach results in MR input functions which are very similar in shape and transit time to the reference standard PET input functions (Fig. 5). The myocardial compartment TAC and TIC closely match in terms of wash-in, however the MR TIC can be observed to have a longer transit time than the radiotracer (Fig. 5, part C). We propose that this may be due to the higher particle mass and viscosity of the GBCA than the radiotracer.

One of the main benefits of the phantom model is its reproducibility. Figure 4 demonstrates that repeat acquisitions at the same $P_{T}$ give similar TAC and TIC. Upon calculation of perfusion via PET (Table 1 ), $K_{1}$ values when performed with independent repeat acquisitions produce values in the range of $1.2 \%$ to $7.5 \%$ of each other. A similar repeatability is shown in MR data with a repeatability of $0.6 \%-13 \%$ for $h(t=0)$. PET measurements at $P_{T}=1 \mathrm{ml} / \mathrm{g} / \mathrm{min}$ showed an overestimation of $P_{T}$ by $16 \%$, however the $P_{T}$ rates of 2, 3, 4 and $5 \mathrm{ml} / \mathrm{g} / \mathrm{min}$ were accurate to $K_{1}$ values to within a maximum of $2.65 \%$, indicating good precision for repeated measurements and also a good accuracy to $P_{T}$ values above $1 \mathrm{ml} / \mathrm{g} / \mathrm{min}$. Inaccuracies in the true measurement of $P_{T}=1 \mathrm{ml} /$ $\mathrm{g} / \mathrm{min}$ due to physical accuracy of the roller pumps may account for the larger differences at this value of $P_{T}$, and will be investigated in the next generation of the phantom currently under development. Although $h(t=0)$ values represent relative perfusion measurement and were not scaled to represent absolute perfusion units, their relationship to $P_{T}$ and $K_{1}$ can be clearly observed in Fig. 6, whereby a linear relationship was found between $h(t=0)$ and $K_{1}$, as well as $h(t=0)$ and $P_{T}$. Another potential advantage of the approach is the possibility to address differences in the way images are acquired and modality-specific artifacts, such as saturation effects in MR or attenuation correction in PET.

Total analysis time of each series of PET images was approximately $30 \mathrm{~min}$, and MR images were approximately 5-10 min. Owing to the geometrical differences between phantom and patient images, semi-automated PET analysis software could not be used. 
Furthermore, PET images were rebinned into short frames of $3 \mathrm{~s}$ because of the rapid transit of the radiotracer in water. In clinical image we expect a lower amount of data and frames to analyse.

Attenuation correction is a major issue in clinical PET-MR imaging and the focus of much research (Mehranian \& Zaidi, 2015). In this work, we utilised GBCA for the bolus injection, and in clinical studies the concentration used would be far higher. Previous work by our group has shown that despite large concentrations of GBCA up to $65 \mathrm{mM}$ (presenting the scenario of GBCA bolus in the left ventricle simultaneously with the PET radiotracer), the effect of attenuation of gamma photons by GBCA on quantified activity concentration $(\mathrm{kBq} / \mathrm{ml})$ in the final reconstructed images is less than $5 \%$ when compared to no GBCA present (O'Doherty \& Schleyer, 2017).

We believe that by providing a standardized setup and known perfusion rates, results, claims and hypotheses from clinical studies can be further investigated. For example, in a recent study by our group, MFR of 41 patients calculated from independent CMR and PET scans have been shown to correlate well, however absolute CMR perfusion at stress and rest correlated weakly and were positively biased compared to their PET counterparts (Morton et al., 2012). This may indicate that errors in quantification have a similar effect on stress and rest perfusion MBF values but are cancelled by calculation of the MFR. Future experiments could verify this finding by the exclusion of physiological variation. The phantom also allows comparison of kinetic models given the known ground truth of perfusion rates, and the potential for development of new hybrid kinetic models employing both PET and MR data.

Knowledge of the relationship between $P_{T}, K_{1}$ and $h(t=0)$ may allow the creation of a modality-specific calibration curves. Particularly in the case of perfusion MR, this could allow converting the results of the deconvolution operation from seconds ${ }^{-1}$ to $\mathrm{ml} / \mathrm{g} / \mathrm{min}$ of perfusion. This approach could prove of value as a substitute for current approaches based on constraining the deconvolution operation (Zarinabad et al., 2013; Hautvast et al., 2012). This may lead to an improvement in the correlation between absolute MBF values measured with MR and PET, which was shown to be suboptimal in comparison with MPR values in previous studies (Morton et al., 2012).

\section{Limitations}

The use of the phantom in this work for simultaneous PET-MR acquisitions as a surrogate for clinical acquisitions presents some fundamental limitations. Despite the fact that the TIC and TAC curves are similar in appearance for this simplified phantom study, it may not be the case for clinical studies in a human cohort due to the mechanism of transport of radiotracer (intracellular) and GBCA (extracellular). Therefore the phantom study serves to provide preliminary investigation into the standardized comparison between PET and MR perfusion values in a controlled simulation. The phantom model used in our experiments is not able to capture the broad range of body structures and physiological states that may be present in a clinical setting and as such represents an oversimplification of the cardiovascular system which cannot detail true myocardial diffusion or radiotracer uptake. Furthermore, despite a good correlation between PET and MR perfusion, there remain fundamental differences between the calculation methodology between the MR model-independent deconvolution approach (leading to a parameter related to perfusion) 
and perfusion as calculated from a single compartment PET model. As is the mechanism with PET radiotracers, no separate tissue compartment exists, for example one with welldefined mechanical properties such as a membrane. Therefore true intracellular uptake cannot be simulated, only allowing non-circulating extravascular transfer of tracer. Strategies would be required in order to simulate the kinetics of perfusion tracers that undergo metabolic processes such as $\left[{ }^{13} \mathrm{~N}\right] \mathrm{NH}_{3}$ or $\left[{ }^{18} \mathrm{~F}\right]$ flurpiridaz. Efforts should be made to create myocardial compartments within the phantom, which would allow a more accurate approach to kinetic modeling. Furthermore, we performed only 1 repeat acquisition of each $P_{T}$ in this feasibility study, further repeat measurements would allow the calculation of a repeatability coefficient for both the PET and MR datasets.

The current phantom model setup is unable to reproduce the multiple sources of image artifacts in PET-MR such as the effects of motion due to respiratory or cardiac contraction. Thus the phantom allows an environment free from these potentially confounding effects focusing only on the assessment of the perfusion dynamics within the cardiac compartments. However, translation of calibrations from the phantom to the clinical setting should be treated with caution. Confounding factors from clinical data may include the use of respiratory correction via importing an average cine CT or using MR-based navigators (Ouyang et al., 2013) or employing MR motion-field based cardiac motion correction employed in PET reconstruction (Huang et al., 2015). Efforts to apply these techniques specifically to quantitative dynamic PET-MR cardiology are in their infancy, although some techniques are currently under development for static imaging (Nensa et al., 2013; Vontobel et al., 2015).

\section{Conclusion}

We have performed a feasibility study of the first simultaneous PET-MR acquisitions from a dynamic cardiac perfusion phantom, showing similar first-pass dynamics of both the PET and MR contrast agents. We have described the resulting simultaneous traces, showed initial repeatability of the phantom studies and also demonstrated a correlation between perfusion quantification of the PET time-activity traces using a kinetic model, relative MR perfusion using a deconvolution model and the true manually set myocardial perfusion rate. The phantom shows potential for improving standardisation of perfusion measurements, analysis routines, development of imaging protocols and potential calibration of MR perfusion values. We have also described the major limitations of the system, detailing how these phantom studies are an important stepping stone allowing investigation of sequence development/comparison and kinetic model development in both PET and MR modalities.

\footnotetext{
Abbreviations

CAD: Coronary artery disease; CMR: Cardiac magnetic resonance; GBCA: Gadolinium based contrast agent; MFR: Myocardial flow reserve; MPI: Myocardial perfusion imaging; PET-MR: Positron emission tomography \& magnetic resonance

Funded by the National Institute for Health Research (NIHR) Biomedical Research Centre award to Guy's \& St Thomas' NHS Foundation Trust in partnership with King's College London and by the NIHR Healthcare Technology Co-operative for Cardiovascular Disease at Guy's and St Thomas' Foundation Trust. Funded by the Wellcome Trust and EPSRC under grant number WT 088641/Z/09/Z.

Funded by King's College London and UCL Comprehensive Cancer Imaging Centre and by the CRUK and EPSRC in association with the MRC and DoH (England).

Funded by the British Heart Foundation award RE/08/003.

The views expressed are those of the author(s) and not necessarily those of the NHS, the NIHR, the DoH, EPSRC or Wellcome Trust.
} 
Availability of data and materials

Data is available from the corresponding authors on a reasonable request.

\section{Authors' contributions}

JOD, ES, MSN, PKM and AC designed the study. JOD, AC and ES carried out phantom preparations, acquisitions and reconstructions. JOD, AC and PS analyzed and interpreted the resulting data. JOD, MSN and AC drafted the manuscript, which was revised by all authors. All authors approved the final manuscript.

\section{Ethics approval and consent to participate}

Not applicable.

\section{Consent for publication}

Not applicable.

\section{Competing interests}

The authors declare that they have no competing interests.

\section{Publisher's Note}

Springer Nature remains neutral with regard to jurisdictional claims in published maps and institutional affiliations.

\section{Author details}

'Division of Imaging Sciences and Biomedical Engineering, PET Imaging Centre, King's College London, St. Thomas' Hospital, 1st Floor Lambeth Wing, St Thomas' Hospital, London SE1 7EH, UK. 'Division of Imaging Sciences, King's College London, Wellcome Trust/EPSRC Medical Engineering Centre, St. Thomas' Hospital, London, UK. ${ }^{3}$ Bristol Heart Institute, Bristol, UK. ${ }^{4}$ Siemens Healthcare Limited, Frimley, Camberley, UK. ${ }^{5}$ Department of Cardiology, Guy's and St Thomas' NHS Foundation Trust, London, UK.

Received: 12 April 2017 Accepted: 1 September 2017

Published online: 12 October 2017

\section{References}

Bengel FM, Higuchi T, Javadi MS, Lautamaki R (2009) Cardiac positron emission tomography. J Am Coll Cardiol 54(1):1-15 Chiribiri A, Schuster A, Ishida M, Hautvast G, Zarinabad N, Morton G et al (2013) Perfusion phantom: An efficient and reproducible method to simulate myocardial first-pass perfusion measurements with cardiovascular magnetic resonance. Magn Reson Med 69(3):698-707

Di Carli MF, Dorbala S, Meserve J, El Fakhri G, Sitek A, Moore SC (2007) Clinical myocardial perfusion PET/CT. J Nucl Med 48(5):783-793

Fihn SD, Gardin JM, Abrams J, Berra K, Blankenship JC, Dallas AP et al (2012) 2012 ACCF/AHA/ACP/AATS/PCNA/SCAI/ STS Guideline for the diagnosis and management of patients with stable ischemic heart disease: a report of the American College of Cardiology Foundation/American Heart Association Task Force on Practice Guidelines, and the American College of Physicians, American Association for Thoracic Surgery, Preventive Cardiovascular Nurses Association, Society for Cardiovascular Angiography and Interventions, and Society of Thoracic Surgeons. J Am Coll Cardiol 60(24):e44-e164

Gerber BL (2012) Quantification of myocardial perfusion and myocardial perfusion reserve by positron emission tomography and cardiovascular magnetic resonance imaging. J Am Coll Cardiol 60(16):1556-1557

Hautvast G, Chiribiri A, Zarinabad N, Schuster A, Breeuwer M, Nagel E (2012) Myocardial blood flow quantification from MRI by deconvolution using an exponential approximation basis. IEEE Trans Biomed Eng 59(7):2060-2067

Huang C, Petibon Y, Ouyang J, Reese TG, Ahlman MA, Bluemke DA et al (2015) Accelerated acquisition of tagged MR for cardiac motion correction in simultaneous PET-MR: phantom and patient studies. Med Phys 42(2):1087-1097

Ishida M, Schuster A, Morton G, Chiribiri A, Hussain S, Paul M et al (2011) Development of a universal dual-bolus injection scheme for the quantitative assessment of myocardial perfusion cardiovascular magnetic resonance. J Cardiovasc Magn Reson 13:28

Jerosch-Herold M (2010) Quantification of myocardial perfusion by cardiovascular magnetic resonance. J Cardiovasc Magn Reson 12:57

Jerosch-Herold M, Wilke N, Stillman AE, Wilson RF (1998) Magnetic resonance quantification of the myocardial perfusion reserve with a Fermi function model for constrained deconvolution. Med Phys 25(1):73

Kero T, Nordstrom J, Harms HJ, Sorensen J, Ahlstrom H, Lubberink M (2017) Quantitative myocardial blood flow imaging with integrated time-of-flight PET-MR. EJNMMI Phys 4(1):1

Martinez-Moller A, Souvatzoglou M, Delso G, Bundschuh RA, Chefd'hotel C, Ziegler SI et al (2009) Tissue classification as a potential approach for attenuation correction in whole-body PET/MRI: evaluation with PET/CT data. J Nucl Med 50(4):520-526

Mehranian A, Zaidi H (2015) Impact of Time-of-Flight PET on Quantification Errors in MR Imaging-Based Attenuation Correction. J Nucl Med 56(4):635-641

Morton G, Chiribiri A, Ishida M, Hussain ST, Schuster A, Indermuehle A et al (2012) Quantification of absolute myocardial perfusion in patients with coronary artery disease: comparison between cardiovascular magnetic resonance and positron emission tomography. J Am Coll Cardiol 60(16):1546-1555

Nensa F, Poeppel TD, Beiderwellen K, Schelhorn J, Mahabadi AA, Erbel R et al (2013) Hybrid PET/MR imaging of the heart: Feasibility and Initial Results. Radiology 268(2):366-373

Nensa F, Schlosser T (2014) Cardiovascular hybrid imaging using PET/MRI. Rofo 186(12):1094-1101 
Nensa F, Tezgah E, Poeppel TD, Jensen CJ, Schelhorn J, Kohler J et al (2015) Integrated 18F-FDG PET/MR imaging in the assessment of cardiac masses: a pilot study. J Nucl Med 56(2):255-260

O'Doherty J, Sammut E, Schleyer P, Stirling J, Nazir S, Marsden PK, et al. Simultaneous PET-MR first-pass perfusion imaging using a novel cardiac perfusion phantom. European Association of Nuclear Medicine Annual Meeting; Barcelona, Spain 2016

O'Doherty J, Schleyer P (2017) The effect of gadolinium-based MR contrast agents on PET attenuation coefficients and quantification during simultaneous dynamic PET-MR for cardiac studies. EJNMMI Phys. 4(4):1-10

O'Doherty J, Schleyer P, Pike L, Marsden P (2014) Effect of scanner dead time on kinetic parameters determined from image derived input functions in 13N cardiac PET. J Nucl Med 55(supplement 1):605

Otton J, Morton G, Schuster A, Bigalke B, Marano R, Olivotti L et al (2013) A direct comparison of the sensitivity of CT and MR cardiac perfusion using a myocardial perfusion phantom. J Cardiovasc Comput Tomogr 7(2):117-124

Ouyang J, Li Q, El Fakhri G (2013) Magnetic resonance-based motion correction for positron emission tomography imaging. Semin Nucl Med 43(1):60-67

Patel AR, Antkowiak PF, Nandalur KR, West AM, Salerno M, Arora V et al (2010) Assessment of advanced coronary artery disease: advantages of quantitative cardiac magnetic resonance perfusion analysis. J Am Coll Cardiol 56(7):561-569

Petibon Y, Ouyang J, Zhu X, Huang C, Reese TG, Chun SY et al (2013) Cardiac motion compensation and resolution modeling in simultaneous PET-MR: a cardiac lesion detection study. Phys Med Biol 58(7):2085-2102

Ratib O, Nkoulou R (2014) Potential Applications of PET/MR Imaging in Cardiology. J Nucl Med 55(Supplement 2):40S-46S

Ratib O, Nkoulou R, Schwaiger M (2013) Cardiovascular clinical applications of PET/MRI. Clin Transl Imaging 1(1):65-71

Renaud JM, Yip K, Guimond J, Trottier M, Pibarot P, Turcotte E, et al (2017). Characterization of 3D PET systems for accurate quantification of myocardial blood flow. J Nucl Med 58(1):103-109

Rischpler C, Langwieser N, Souvatzoglou M, Batrice A, van Marwick S, Snajberk J et al (2015) PET/MRI early after myocardial infarction: evaluation of viability with late gadolinium enhancement transmurality vs. 18F-FDG uptake. Eur Heart J Cardiovasc Imaging 16(6):661-669

Rischpler C, Nekolla SG, Dregely I, Schwaiger M (2013) Hybrid PET/MR imaging of the heart: potential, initial experiences, and future prospects. J Nucl Med 54(3):402-415

Rischpler C, Nekolla SG, Kunze KP, Schwaiger M (2015) PET/MRI of the heart. Semin Nucl Med 45(3):234-247

Schneider S, Batrice A, Rischpler C, Eiber M, Ibrahim T, Nekolla SG (2014) Utility of multimodal cardiac imaging with PET/MRI in cardiac sarcoidosis: implications for diagnosis, monitoring and treatment. Eur Heart J 35(5):312

Schuster A, Sinclair M, Zarinabad N, Ishida M, van den Wijngaard JP, Paul M et al (2013) A quantitative high resolution assessment of myocardial blood flow from contrast-enhanced first-pass magnetic resonance perfusion imaging: microsphere validation in a magnetic resonance compatible free beating explanted pig heart model. J Cardiovasc Magn Reson 15(Suppl 1):E19

Task Force M, Montalescot G, Sechtem U, Achenbach S, Andreotti F, Arden C et al (2013) 2013 ESC guidelines on the management of stable coronary artery disease: the Task Force on the management of stable coronary artery disease of the European Society of Cardiology. Eur Heart J 34(38):2949-3003

Vontobel J, Liga R, Possner M, Clerc OF, Mikulicic F, Veit-Haibach P et al (2015) MR-based attenuation correction for cardiac FDG PET on a hybrid PET/MRI scanner: comparison with standard CT attenuation correction. Eur I Nucl Med Mol Imaging 42(10):1574-1580

Zarinabad N, Chiribiri A, Hautvast G, Shuster A, Sinclair M, van den Wijngaard JP et al (2013) Modelling Parameter Role on Accuracy of Cardiac Perfusion Quantification. In: Ourselin S, Rueckert D, Smith N (eds) Functional Imaging and Modeling of the Heart. Springer-Verlag, Heidelberg

Zarinabad N, Chiribiri A, Hautvast GL, Ishida M, Schuster A, Cvetkovic Z et al (2012) Voxel-wise quantification of myocardial perfusion by cardiac magnetic resonance. Feasibility and methods comparison. Magn Reson Med 68(6): 1994-2004

Zhang HS, Rischpler C, Langweiser N, Hayes C, Batrice A, Ibrahim T et al (2013) Simultaneous Measurement of Myocardial Perfusion by Dynamic Contrast Enhancement MR and Ammonia PET. Proc Int Soc Magn Reson Med Sci Meet Exhib Int Soc Magn Reson Med Sci Meet Exhib 21:0576

\section{Submit your manuscript to a SpringerOpen ${ }^{\odot}$} journal and benefit from:

- Convenient online submission

Rigorous peer review

Open access: articles freely available online

- High visibility within the field

- Retaining the copyright to your article

Submit your next manuscript at $\boldsymbol{s p r i n g e r o p e n . c o m ~}$ 